\title{
Effect of Optic Nerve Stimulation on the Intraretinal ERG of the Cat
}

\author{
By \\ Kyoji Tasaki, Allen C. Norton* and Yoshiro Fukada ${ }^{+}$ \\ From The Physiological Laboratory of Prof. K. Motokawa \\ Tohoku University School of Medicine, Sendai
}

(Received for publication, September 1, 1962)

\section{INTRODUCTION}

From the observation that stimulation of the optic nerve had no influence on the ERG, Granit and Helme ${ }^{1)}$ concluded that the ganglion cells make no essential contribution to the generation of the ERG. Recently, however, Jacobson and Gestring ${ }^{2,3)}$ have demonstrated that repetitive stimulation of the bulbar reticular formation caused a marked reduction of the amplitude of the ERG of the cat and monkey; this effect was attributed to the activity of centrifugal fibers in the optic nerve.

The present paper is a preliminary report attempting to resolve such discrepant results concerning efferent optic nerve influence on the ERG. The intraretinal ERG was used to obtain finer details of the retinal potentials.

\section{METHOD}

The experimental animals were cats. Surgery was performed under ether anesthesia. The eye was prepared for insertion of microelectrodes as described by Brown and Wiesel ${ }^{4}$. The ipsilateral hemisphere was exposed and gently lifted to allow placement of the stimulating electrodes (silver wires) by visual control. Following surgery, the wound margins were infiltrated with procain, the pupil was dilated with atropine, Flaxedil was injected and artificial respiration was begun.

The recording electrodes were insulated stainless steel (less than $1 \mu$ tip diameter, see Brown and Tasaki $\left.{ }^{5}\right)$, and the indifferent electrode was a spiral of $\mathrm{Ag}-\mathrm{AgCl}$ wire in the animal's mouth. Recordings were made with a high gain DC amplifier with a cathode follower input stage. Light stimuli were provided by a Sylvania glow modulator tube (R1131C) which was activated by an electronic

田崎 京二, 深 田 芳 郎

* This investigation was carried out during the tenure of a postdoctoral fellowship from the National Institute of Mental Health, United States Public Health Service.

+ Technical Research Laboratories, Japan Broadcasting Corporation (NHK) 
quare pulse generator. Before recordings were made the animal was darkdapted for about 10 minutes. A constant adaptation level was maintained by resenting one light flash ( $0.5 \mathrm{sec}$. duration) every 20 seconds. The intensity of he optic nerve stimulation was adjusted to be supramaximal with respect to he antidromic response recorded at the optic disk.

\section{RESULTS}

The ERG recorded from a microelectrode in the vitreous body had the same onfiguration as that recorded from the cornea but was much larger in amplitude. $n$ agreement with the observation of Granit and Helme ${ }^{1)}$, stimulation of the iptic nerve failed to modify the ERG, even over a wide range of stimulus variibles (intensity, pulse width, and frequency). Fig. 1 shows the vitreous ERG luring optic nerve stimulation on the right and the control on the left. The nly noticeable difference is the presence of the shock artifacts in the former ecord.
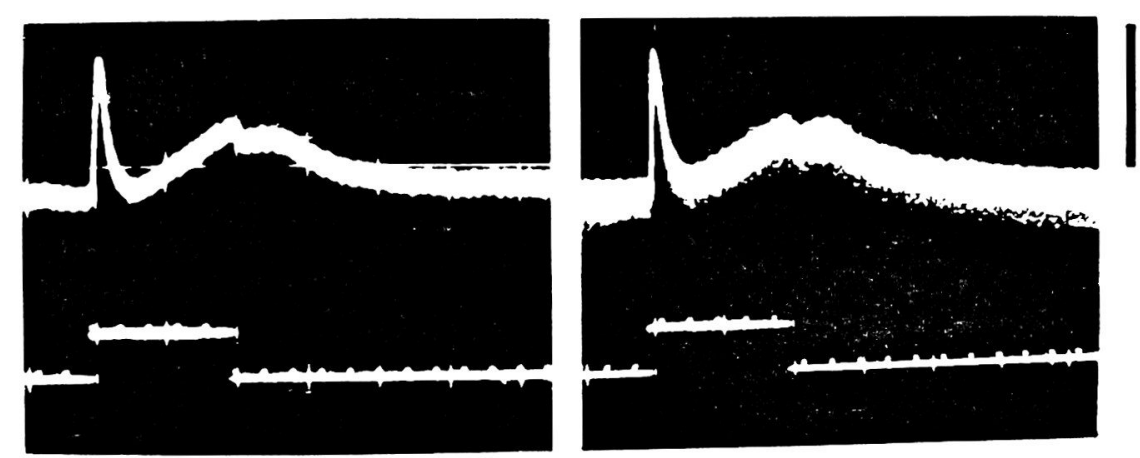

Fig. 1. Effect of optic nerve stimulation on the vitreous ERG. Left, control response to single flash. Right, response to flash during stimulation (200 cps) of the optic nerve; distorted trace shows stimulus artifacts. Lower trace shows light stimulus. Vertical bar, $0.5 \mathrm{mV}$. Time mark, $10 \mathrm{cps}$.

When the microelectrode was inserted through the retina, a consistent sequence of responses was observed following illumination (see the right records of Fig. 9 in Brown and Wiesel $\left.{ }^{6}\right)$. The amplitude of the intraretinal ERG is larger ihan either the corneal or the vitreous ERG. The polarities of the a- and b-waves $\mathrm{n}$ the intraretinal ERG are the opposite of those seen in the vitreous (or corneal) ERG, but the c-wave maintains the same polarity despite changes in the site of ;he active electrode. Among the various components of the intraretinal ERG, ;he c-wave was found to be most suitable for studying the effect of optic nerve stimulation, because it has the longest time course and was least obscured by the stimulus artifacts. Thus the present study reports principally the effect of optic 

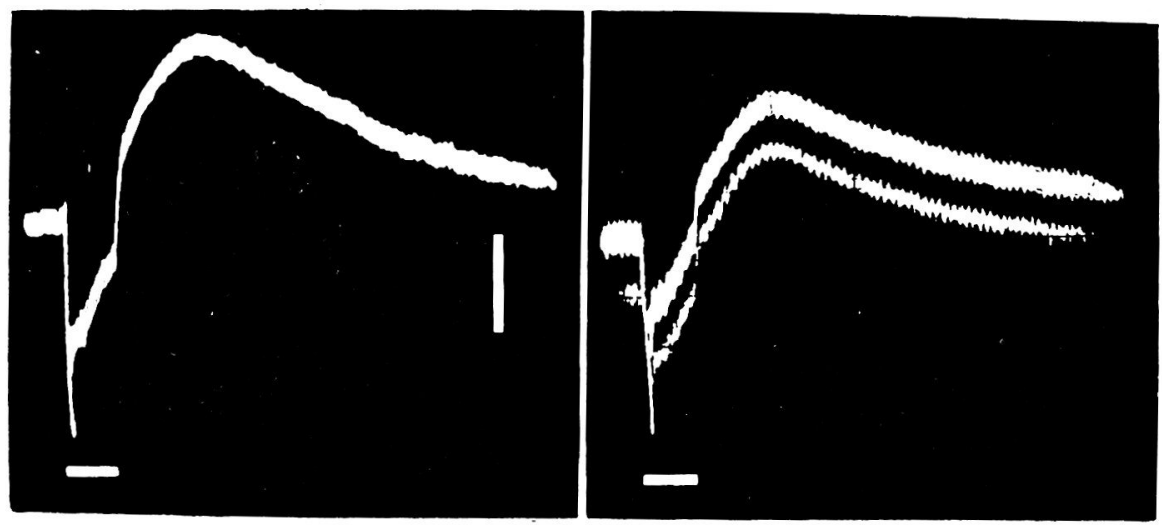

Fig. 2. Effect of optic nerve stimulation on the intraretinal FRG. Electrode near Bruch's membrane. Left, control response to single flash. Right, response to flash during stimulation (200 cps). Horizontal bars, 0.5 sec. light stimulus mark. Vertical bar, $1 \mathrm{mV}$.

nerve stimulation on the intraretinal c-wave. The left record of Fig. 2 was obtained from a microelectrode placed immediately adjacent to the retinal side of Bruch's membrane; at this level the amplitude of the c-wave is maximum. The right record in Fig. 2 shows the reduction of amplitude of the c-wave during repetitive stimulation (200 cps) of the optic nerve. In this case the amplitude of the c-wave was reduced by about $25 \%$. Fig. 3 shows that the spontaneous variability of the c-wave is considerably less than the reduction in amplitude induced by optic nerve stimulation.

In a few cases similar effects of optic nerve stimulation were seen for other components of the intraretinal ERG and the intraretinal graded photic response (S-potential). In still other cases, the opposite effect was observed, immediately following optic nerve stimulation the amplitude of the c-wave was augmented and then gradually decreased to, or even below, the control level. The present study did not persue the details of such atypical observations.

\section{DISCUSSION}

It is clear, at least for the intraretinal c-wave, that repetitive stimulation of the optic nerve causes a reduction of the ERG amplitude. This observation could be explained either by an alteration in retinal circulation or by centrifugal influence. If optic nerve stimulation alters only the retinal circulation, the suprression of the ERG is merely a secondary effect. This possibility was investigated by temporarily clamping both carotid arteries. Under such conditions the intraretinal b-wave was observed to decrease in amplitude, but the c-wave maintained its same amplitude during many minutes of impaired circulation. On the other hand, considerable evidence has accumulated concerning centrifugal 


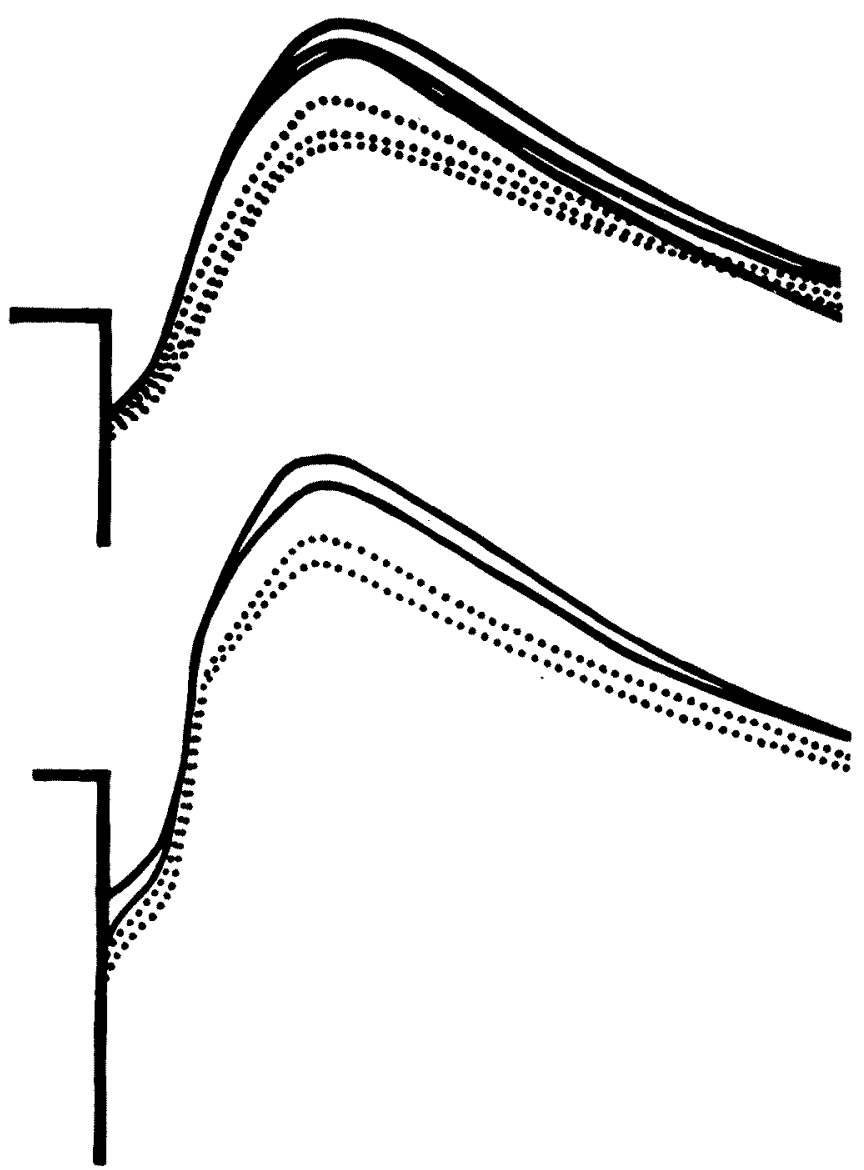

Fig. 3. Variability of the intraretinal c-wave amplitude. Solid traces, control; dotted traces, during stimulation. Upper and lower sets of superimposed traces taken from different animals.

control of retinal events. Centrifugal influence on the spike discharge of the retinal ganglion cells, for example, has been suggested by Motokawa and Ebe?), Granit ${ }^{8)}$, and Dodt. ${ }^{9}$ It has also been reported that centrifugal effects on retinal functions can be detected by recording the ERG as an indicator. ${ }^{10-12}$ ) Recently, Jacobson and Gestring ${ }^{2,3}$ ) have demonstrated the section of the optic nerve abolishes the effects of drugs on the ERG in the cat and monkey. Furthermore, $\mathrm{Abe}^{13}$ ) found that section of the optic nerve caused a remarkable increase in the rabbit ERG, the maximum increase in amplitude of the b-wave appearing at 3 to 6 hours after sectioning. The observations of Jacobson and Gestring ${ }^{2,3}$ and $A \mathrm{Ab}^{13}$ ) could well be explained as indicating an inhibitory influence of centrifugal fibers in the optic nerve. Thus it seems reasonable to interpret the 
present experimental results as arising from inhibitory activity of efferent fibers in the optic nerve, tentatively rejecting the possibility of an alteration of the retinal circulation.

Nonetheless, several questions still remain: 1) whether the rare observation of a facilitatory effect of optic nerve stimulation might indicate the existence of excitatory fibers, 2) why the inhibitory effect of optic nerve stimulation could be detected only by recording the intraretinal ERG, and 3) whether optic nerve stimulation is also effective on the other components of the ERG. Further experiments will be attempted to answer these questions.

\section{SUMMARY}

The cat intraretinal ERG was recorded with microelectrodes. Repetitive stimulation of the optic nerve reduced the amplitude of the ERG, especially that of the c-wave. This effect on the intraretinal ERG was tentatively attributed to centrifugal fibers in the optic nerve, although the possibility that it was a secondary effect resulting from an alteration of the retinal circulation could not be excluded completely.

The authors express thanks to Prof. K. Motokawa for his helpful discussion and comments during experimentation and preparation of this manuscript.

\section{References}

1) Granit, R. \& Helme, T., J. Neurophysiol., 1939, 2, 556.

2) Jacobson, J.H. \& Gestring, G.F., A.M.A. Arch. Ophthal., 1958, 60, 295.

3) Jacobson, J.H. \& Gestring, G.F., Ann. New York Acad. Sci., 1958, 74, 362.

4) Brown, K.T. \& Wiesel, T.N., J. Physiol., 1959, 149, 537.

5) Brown, K.T. \& Tasaki, K., J. Physiol., 1961, 158, 281.

6) Brown, K.T. \& Wiesel, T. N., J. Physiol., 1961, 158, 229.

7) Motokawa, K. \& Ebe, M., J. Neurophysiol., 1954, 17, 364.

8) Granit, R., J. Neurophysiol., 1955, 18, 388.

9) Dodt E., J. Neurophysiol. 1956, 19, 301.

10) Müller-Limmroth, H.W., Z. Biol., 1954, 107, 216.

11) Marg, E., A.M.A. Arch. Ophthal., 1951, 45, 169.

12) Motokawa, K., Nakagawa D. \& Kohata, T., J. comp. physiol. Psychol., 1956, 49, 398.

13) Abe, N., Tohoku J. Exper. Med., 1962, 78 (to be published). 